\title{
Salud mental durante el confinamiento impuesto en España: efectos de hacer ejercicio y sacar a pasear al perro sobre estado de ánimo, sintomatología depresiva e ideación suicida
}

\section{Mental health during confinement imposed in Spain: Effects of exercising and taking the dog for a walk on mood, depressive symptoms and suicidal ideation}

\author{
Nicolás Sánchez-Álvarez y Darío Bellido-Guillén \\ Universidad de Málaga, España
}

\section{Resumen}

Debido a la urgencia mundial a causa de la COVID-19, la población ha sufrido cambios en su estilo de vida, provocando en muchas ocasiones un gran impacto emocional. Este estudio se llevó a cabo en una muestra de 765 personas en confinamiento impuesto por el marco de medidas durante el estado de alarma en España a partir del 14 de marzo de 2020. Tiene como objetivo profundizar en el impacto emocional causado por el estado de confinamiento domiciliario. Se analizó la influencia de variables como la extensión del confinamiento domiciliario, la realización de ejercicio físico diario y sacar a pasear al perro en los niveles del estado afectivo (afectividad positiva y negativa), la sintomatología depresiva y la ideación suicida. Los resultados multivariantes mostraron una influencia significativa de la extensión del confinamiento sobre la ideación suicida, y destaca el papel del ejercicio diario como potenciador directo del estado de ánimo positivo y como reductor indirecto de la sintomatología depresiva y la ideación suicida. Estos resultados son de utilidad para la planificación de situaciones futuras de confinamiento y el efecto que tiene en la población, así como para la evaluación de los mecanismos de influencia psicológicos en estados de privación de libertad.

\begin{abstract}
Due to the global urgency of COVID-19, the population's lifestyle has changed, often causing a tremendous emotional impact. The purpose of this study, carried out in a sample of 765 people under imposed confinement within the framework of Spain's state of alarm as of March 14, 2020, is to explore in depth the emotional impact caused by these home confinement measures. The influence of variables such as the extension of home confinement, daily physical exercise, and taking the dog out for a walk on the state levels of affectivity (positive and negative), depressive symptomatology, and suicidal ideation was analyzed. The multivariate results showed a significant influence of confinement on suicidal ideation and highlighted the role of daily exercise as a direct enhancer of positive mood and indirect reducer of depressive symptomatology and suicidal ideation. These results are helpful for planning future situations of confinement and its effect on the population and for evaluating the mechanisms of psychological influence in times of deprivation of liberty.
\end{abstract}

Keywords: confinement, COVID-19, mood, depression, suicidal ideation.

Palabras clave: confinamiento, COVID-19, estado de

ánimo, depresión, ideación suicida.

Cómo citar: Sánchez-Álvarez, N., \& Bellido-Guillén, D. (2021). Salud mental durante el confinamiento impuesto en España: efectos de hacer ejercicio y sacar a pasear al perro sobre estado de ánimo, sintomatología depresiva e ideación suicida. Escritos de Psicología - Psychological Writings, 14(2), 73-83. https://doi.org/1024310/espsiescpsi.v14i2.12501

Correspondencia: Nicolás Sánchez Álvarez. Facultad de Psicología. Universidad de Málaga. 29071 Málaga, España. E-mail: nsa@uma.es 


\section{Introducción}

Debido a la urgencia mundial a causa del Covid-19 han surgido numerosos cambios repentinos que pueden afectar a numerosos ámbitos personales. En España a partir del día 14 de marzo alrededor del $40 \%$ de la población estuvo sometida a confinamiento domiciliario (Fariñas, 2020). El estado de confinamiento domiciliario tuvo un gran impacto emocional en la población debido a la privación de libertad y el estrés del aislamiento. Numerosos estudios corroboran que aquellas personas que han sido sometidas a situaciones de confinamiento muestran una prevalencia alta de síntomas depresivos (Hawryluck et al., 2004), irritabilidad (Lee, Chan, Chau, Kwok, \& Kleinman, 2005), afectividad negativa (Lee et al., 2005), estrés (DiGiovanni, Conley, Chiu, \& Zaborski, 2004), malestar emocional (Yoon, Kim, Ko, \& Lee, 2016), insomnio (Lee et al., 2005), síntomas de estrés post-traumático (Reynolds et al., 2008), y ansiedad (Jeong et al., 2016). Asimismo, la duración total del confinamiento correlaciona de manera negativa con la salud mental (Brooks et al., 2020; Hawryluck et al., 2004; Marjanovic, Greenglass, \& Coffey, 2007; Reynolds et al., 2008). Esta relación se amplifica de manera más significativa a partir de los 10 días (Hawryluck et al., 2004). Además, cuarentenas con duración indefinida pueden ser más perjudicial a nivel psicológico que las limitadas a la etapa de incubación del virus (Brooks et al, 2020). Sin embargo, estas evidencias no sugieren que el confinamiento no deba ser usado, puesto que posiblemente, de no usarlo, los efectos psicológicos podrían ser aún más adversos (Brooks et al., 2020). Existen pocos estudios que hayan profundizado en la influencia del confinamiento en el estado de ánimo, y cómo influye en los pensamientos suicidas.

Actualmente, existe un creciente interés sobre la literatura sobre el bienestar percibido (Myers \& Diener, 1995; VanderZee, Buunk, \& Sanderman, 1995). En ese aspecto, la actividad física se engloba dentro de la psicología de la salud (Granados \& Cuéllar, 2018). Existen ciertos factores que pueden ayudar a prevenir el impacto del confinamiento y mejorar el bienestar, uno de estos factores que más representatividad ha tenido durante el confinamiento ha sido la práctica de ejercicio físico. Practicar ejercicio físico aporta beneficios en distintas áreas del bienestar subjetivo (Jiménez, Martínez, Miró, \& Sánchez, 2008), favorece un mejor estado de ánimo, aumenta la salud subjetiva (Biddle, Fox, \& Boutcher, 2000), y aumenta los niveles de autoestima (Huertas et al, 2003). Por otro lado, la práctica de deportes y ejercicio físico mejora el afrontamiento del estrés, disminuye la depresión clínica (Lawlor \& Hopker, 2001) y la ansiedad (Akandere \& Tekin, 2005; Garnier \& Waysfeld, 1995). Asimismo, la práctica deportiva también tiene un efecto protector sobre el riesgo de suicidio (Alzate Pérez et al., 2013). Sujetos con conductas suicidas presentaron una menor práctica de actividades deportivas (Gonzá- lezForteza, Mariño, Mondragón, \& Medina-Mora, 2000).

Por otro lado, en el contexto de confinamiento domiciliario, las autoridades permitieron una serie de conductas fuera del domicilio. Una de ellas consistió en la posibilidad de salir del domicilio para sacar a pasear al perro (Real Decreto 463/2020). Esta acción de pasear al perro se concibió como una de las conductas permitidas que servía como evasión del confinamiento domiciliario, lo cual provocó que esta conducta se reiterara (Oria et al., 2020). Sacar a pasear al perro tenía una función de cobertura legal para posibilitar la salida del domicilio con el fin de aliviar la presión de la privación de libertad. La interacción entre humanos y animales favorece la salud a nivel físico, psicológico, y social (Balazote, 1999; Gutiérrez, Granados, \& Piar, 2007). Asimismo, sacar a pasear al perro promueve una mejora en el bienestar subjetivo, en ansiedad y depresión, contribuyendo de esta forman a una satisfacción general (Balazote, 1999; Wilson, 1998). Relacionarse con animales favorece la atención, el sentido del humor, la interacción social, el contacto físico, la independencia, el sentimiento de valor y la motivación (Gutiérrez et al., 2007). Por consiguiente, sacar a pasear al perro puede ser un factor de protección de la depresión y la sensación de soledad, además que durante el confinamiento domiciliario tuvo una función evasiva de las restricciones de libertad.

Por otro lado, además de analizar los factores confinamiento, ejercicio físico diario y sacar a pasear al perro, el suicidio es una problemática de índole muy relevante, independientemente del contexto histórico. El suicido es de suma importancia dentro de la salud pública y por lo tanto es imprescindible sensibilizar a la población y otorgar un estatus más accesible a la prevención de ésta (World Health Organization - WHO, 2014). Analizar el fenómeno del suicidio conlleva una gran complejidad dado que es el resultado de numerosos factores, entre los que podemos encontrar los psicológicos, sociales, ambientales, familiares, interpersonales, biológicos e incluso enfermedad mental (De la Torre, Costillas, \& Sánchez-Álvarez, 2016). La conducta de suicidio consumado constituye una de las causas de muerte entre las personas con depresión, bipolaridad, esquizofrenia, y sujetos con problemas vinculados al consumo de sustancias (Foster, Gillespie, \& McClelland, 1997). Asimismo, existen otros muchos factores de riesgo en el fenómeno del suicidio, como pueden ser los problemas familiares (Guibert, Reyes, \& Torres, 2001), legales, laborales, dificultades socioeconómicas (De la Torre et al., 2016; 
Thacore \& Varma, 2000), el abuso de sustancias, aislamiento social, antecedentes de maltrato físico y/o sexual en la etapa infantil (Brown, Cohen, Johnson, \& Smailes, 1999; Qin, Agerbo, \& Mortensen, 2003), trastornos con síntomas somáticos (De Leo et al, 1999), trastornos psicológicos (Arsenault-Lapierre, Kim, \& Turecki, 2004; Cavanagh, Carson, Sharpe, \& Lawrie, 2003; Foster et al, 1997), e intentos de suicidios (Castro-Rueda, Martínez-Villalba, Camacho, \& Rueda-Jaimes, 2010). Además, se encontró relaciones significativas entre el estado de ánimo, las ideaciones suicidas y la anhedonia (Siabato Macías, Forero Mendoza, \& Salamanca Camargo, 2017).

El confinamiento tiene efecto sobre el estado de ánimo negativo (Lee et al., 2005). Este estado de ánimo se relaciona a su vez con la sintomatología depresiva (Siabato Macías et al., 2017). Del mismo modo, esta sintomatología tiene una relación directa con el riesgo de suicidio (Rosselló \& Hernández, 2004). Por otro lado, existen factores protectores que favorecen a la disminución de la probabilidad del suicidio y actúan de amortiguador ante situaciones aversivas y estresantes (Seligman, \& Csikszentmihalyi, 2000) como puede ser la realización de actividades físicas (Alzate Pérez et al., 2013).

En consecuencia, de lo anteriormente expuesto y poniendo de manifiesto la escasa literatura sobre el confinamiento y la relación con diferentes variables, el presente estudio pretendió conocer si las variables mencionadas estaban relacionadas con el confinamiento de manera significativa. En concreto, se estudió cómo el confinamiento, el ejercicio físico y sacar a pasear al perro influyeron sobre el estado de ánimo, la sintomatología depresiva y la ideación suicida. A su vez, si el estado de ánimo influyó en la sintomatología depresiva y ésta a su vez sobre la ideación suicida. La Figura 1 muestra la relación que se propone entre estas variables.

Figura 1

Modelo conceptual de relación entre las variables

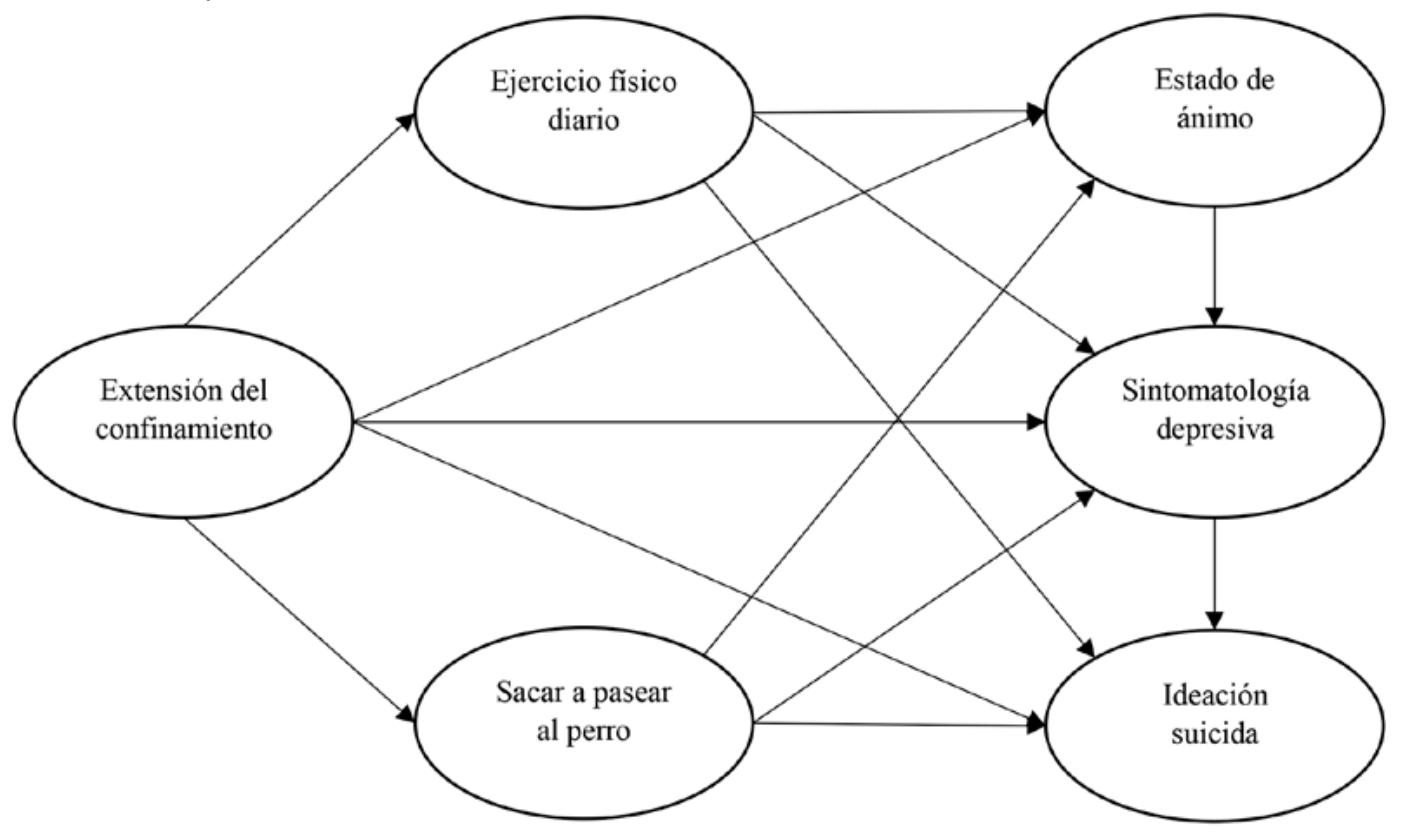

Por tanto, el objetivo general de este estudio fue examinar la realización de ejercicio físico diario y sacar a pasear al perro en el estado de ánimo, la sintomatología depresiva y la ideación suicida durante el confinamiento domiciliario impuesto por el Gobierno de España en el primer estado de alarma entre el 14 de marzo y el 20 de junio de 2020. Los objetivos específicos fueron examinar las diferencias del estado de ánimo entre los participantes que realizaron la conducta de sacar a pasear al perro durante el confinamiento domiciliario (O1); analizar las diferencias del estado de ánimo entre los participantes que realizaron ejercicio físico diario durante el confinamiento domiciliario (O2); comprobar la existencia de relaciones entre la extensión del confinamiento domiciliario, sacar a pasear al perro, la práctica de ejercicio físico diario, el estado de ánimo, la sintomatología depresiva y la ideación suicida (O3); y analizar la interacción multivariante y la relación de la realización de prácticas de ejercicio físico y sacar a pasear al perro sobre el estado anímico durante el confinamiento domiciliario (O4). En base a estudios previos se hipotetizó que los participantes del estudio que hubiesen realizado la conducta de sacar a pasear al perro durante el confinamiento domiciliario tendrían mejores indicadores de estado de ánimo (H1). De forma similar, se estimó que los participantes del estudio que hubiesen realizado ejercicio físico diario durante el confinamiento domiciliario tendrían mejores indicadores de estado de ánimo (H2). Asimismo, 
se pronosticó la existencia de relaciones significativas entre la extensión del confinamiento domiciliario, sacar a pasear al perro, la práctica de ejercicio físico diario, el estado de ánimo, la sintomatología depresiva y la ideación suicida (H3). Por último, se hipotetizó que la realización de ejercicio físico diario y sacar a pasear al perro durante la extensión del confinamiento mejoraría el estado de ánimo y disminuye la sintomatología depresiva y la ideación suicida $(\mathrm{H} 4)$.

\section{Participantes}

\section{Método}

Para esta investigación se evaluaron a sujetos mayores de 18 años, de nacionalidad española y que estuviesen sometidos a la situación de confinamiento domiciliario en España a partir del 14 de marzo. Obteniendo una muestra total de 765 adultos (33,1\% hombres y $66,9 \%$ mujeres). La edad media fue de 31,2 años y la desviación típica de 11,6. La edad mínima de los participantes fue de 18 años y la edad máxima de 68 años. En cuanto al nivel educativo, el 3,4\% de las personas tenían formación en educación primaria, el $19 \%$ en formación profesional, el 11,9\% en educación secundaria, el 3,9\% en bachillerato y el $61,8 \%$ en formación universitaria. En cuanto el estado civil, el $21,4 \%$ de las personas estaban casados/as, el 12,9\% eran pareja de hecho, el 4,2\% estaban separados/as, el $61 \%$ solteros/as y $0,4 \%$ eran viudos/as. Se descartaron 23 participantes por residir fuera de España en el momento del estado de confinamiento domiciliario o ser de nacionalidad extranjera.

\section{Instrumentos}

Las variables relativas al contexto del confinamiento domiciliario fueron recopiladas a través de preguntas como sexo y edad, nivel educativo, estado civil, nacionalidad, país de residencia, la extensión del confinamiento en días (diferencia entre el primer día de confinamiento domiciliario y la fecha de realización de los cuestionarios), realización de ejercicio físico diario (¿Realiza ejercicio físico diario?), y sacar a pasear al perro (¿Saca a pasear al perro a la calle a diario?).

Por otro lado, las variables evaluadas sobre el estado de ánimo fueron la afectividad positiva y negativa, sintomatología depresiva e ideación suicida, todas ellas registradas mediante instrumentos estandarizados descritos a continuación.

La Escala de Afectividad Positiva y Negativa (PANAS; Positive and Negative Affect Schedule) fue desarrollada por Watson, Clark, y Tellegen (1988) con la finalidad de cuantificar tanto el afecto positivo como el negativo. El afecto positivo (PA) hace referencia a personas activas y entusiastas, se registra a través de diez ítems. Un PA alto muestra un nivel pleno de concentración y placentero, en cambio, un nivel bajo de PA muestra letargia y tristeza. Por otro lado, el afecto negativo (NA) se puede ver reflejado en estados de ánimo aversivos, como miedo, culpa, nerviosismo, desprecio e ira, en cambio un bajo nivel de NA muestra sosiego y tranquilidad, y se registra a través de diez ítems. Ambas referidas a dimensiones de estado, no de rasgo (Watson \& Clark, 1984). Tanto el factor positivo como el negativo son fundamentales para entender el bienestar subjetivo (Buz, Pérez-Arechaederra, Fernández-Pulido, \& Urchaga, 2015; Chen, Jing, Hayes, \& Lee, 2013).

La Escala de Depresión, Ansiedad y Estrés (DASS; Depression, Anxiety and Stress Scales) fue creada por Lovibond y Lovibond (1995) con la finalidad de cuantificar los niveles de depresión, ansiedad y estrés. Para este estudio solo se utilizó la dimensión de depresión, evaluada a través de nueve ítems. Presenta buena validez convergente y divergente (Bados, Solanas, \& Andrés, 2005).

El Inventario de Frecuencia de Ideación Suicida (FSII; Frequency of Suicidal Ideation Inventory) fue desarrollada por Chang y Chang (2016) con el objetivo de evaluar la frecuencia de las ideaciones suicidas a través de cinco ítems. Asimismo, es una escala muy valiosa para medir la probabilidad de futuros intentos de suicidio. Igualmente presenta buena validez y fiabilidad siendo una prueba breve (Chang \& Chang, 2016; Sánchez-Álvarez, Extremera-Pacheco, Rey, Chang \& Chang, 2020).

\section{Procedimiento}

Esta investigación se realizó con una muestra de conveniencia de participantes universitarios y comunitarios mayores de 18 años, utilizando la metodología de muestreo reclutada por estudiantes universitarios (siguiendo las pautas proporcionadas por Wheeler, Shanine, Leon, \& Whitman, 2014). Dichos estudiantes entrenados por uno de los autores del estudio, distribuyeron encuestas a los participantes de la universidad y la comunidad mediante enlaces a la batería de instrumentos de "Google Forms" y se compartió a través de las diferentes redes sociales. La recopilación de cuestionarios abarco desde Marzo hasta Mayo de 2020. El cuestionario se realizó de manera individual mediante cualquier dispositivo con acceso a internet y de formato autoaplicado. La realización de esta escala fue voluntaria y de carácter confidencial, los participantes dieron su consentimiento informado mediante la autorización en 
registro online. Esta investigación cuenta con la aprobación del Comité Ético de Experimentación de la Universidad de Málaga (№ registro CEUMA: 93-2020-H).

\section{Análisis estadísticos}

Para realizar el análisis estadístico se utilizó el software IBM SPSS Statistics 25. Los datos sociodemográficos y las variables dependientes se analizaron mediante estadísticas descriptivas. Se aplicó la T-Student para el análisis de las diferencias de medias entre la variable tener perro y la variable realizar ejercicio físico. Para cuantificar la magnitud del tamaño del efecto de las diferencias en las puntuaciones medias se utilizó la $d$ de Cohen (1988), que estima valores próximos a 0,20 como pequeños, en torno a 0,50 como magnitud moderada, y valores alrededor de 0,80 como grandes. Asimismo, se utilizó Pearson para analizar las correlaciones entre las diferentes variables. Para examinar el impacto que tuvo los días de confinamiento, el realizar ejercicio físico a diario y sacar a pasear al perro sobre el estado de ánimo, la sintomatología depresiva, y la ideación suicida (H6), se utilizó un enfoque de análisis de ecuaciones estructurales (SEM) utilizando el software AMOS 20. Para evaluar la bondad de ajuste del modelo se utilizaron Chi-cuadrado $\left(X^{2} / d f\right)$, la aproximación del error cuadrático medio (RMSEA), y el índice de ajuste comparativo (CFI) (Reise, Scheines, Widaman, \& Haviland, 2013).

\section{Resultados}

En primer lugar, se realizó un análisis descriptivo de las variables. Como se puede observar en la Tabla 1 la extensión del confinamiento mostró una media de 25,4 días $(D T=7,95)$, siendo el mínimo 1 día y el máximo 48 días de confinamiento. Para la evaluación de la afectividad positiva se obtuvo una media de 2,84 $(D T=0,84)$, siendo la puntuación mínima 1 y la máxima 5 , mientras que para la afectividad negativa se obtuvo una media de 2,26 $(D T=0,78)$, siendo la puntuación mínima 1 y la máxima 4,8 . En cuanto a la variable sintomatología depresiva se obtuvo una media de $10,70(D T=4,05)$, siendo la puntuación mínima de 7 y la máxima de 28. Por último, en la variable frecuencia de ideación suicida tuvo una media de $6,7(D T=3,05)$ siendo la puntuación mínima de 5 y la máxima de 23 .

Tabla 1

Datos descriptivos de las variables dependientes e independientes

\begin{tabular}{lcccccc}
\hline & Edad & $\begin{array}{c}\text { Extensión } \\
\text { confinamiento }\end{array}$ & Afectividad positiva & Afectividad negativa & $\begin{array}{c}\text { Sintomatología } \\
\text { depresiva }\end{array}$ & Ideación Suicida \\
\hline Media & 31,2 & 25,4 & 2,84 & 2,26 & 10,7 & 6,7 \\
Desviación típica & 11,6 & 7,95 & 0,84 & 0,78 & 4,05 & 3,05 \\
Mínimo & 18 & 1 & 1 & 1 & 7 & 5 \\
Máximo & 68 & 48 & 5 & 4,8 & 28 & 23 \\
\hline
\end{tabular}

En la Tabla 2, la variable sacar a pasear al perro obtuvo una frecuencia de realización de 262 personas $(34,2 \%)$ frente a $503(65,8 \%)$ que no realizaban dicha conducta. En cuanto a la variable ejercicio físico diario se registró una frecuencia de $440(57,5 \%)$ personas que realizaban ejercicio físico a diario frente a $325(42,5 \%)$ que no lo realizaban.

Tabla 2

Datos descriptivos variables dicotómicas

\begin{tabular}{lccc}
\hline & Respuesta & $\mathrm{n}$ & $\%$ \\
\hline \multirow{2}{*}{ Sacar a pasear al perro } & Sí & 262 & $34,2 \%$ \\
& No & 503 & $65,8 \%$ \\
Ejercicio físico diario & Sí & 440 & $57,5 \%$ \\
\hline
\end{tabular}

Los resultados sobre las diferencias de puntuaciones medias entre aquellos participantes que realizaban la conducta de sacar a pasear al perro y aquellos que no, mostraron una ausencia de diferencias significativas en el estado de ánimo. Estos resultados no respaldan la hipótesis inicial (H1) que pronosticaba mejores indicadores de estado de ánimo entre aquellos participantes del estudio que hubiesen realizado la conducta de sacar a pasear al perro durante el confinamiento domiciliario (ver Tabla 3). 
Tabla 3

Diferencias significativas entre personas que realizaban la conducta de sacar a pasear al perro y aquellas que no en las puntuaciones medias

\begin{tabular}{|c|c|c|c|c|c|c|}
\hline & Sacar a pasear al perro & Media & Desviación típica & $T$ & $p$ & $d$ \\
\hline Extensión confinamiento & $\begin{array}{l}\text { Sí } \\
\text { No }\end{array}$ & $\begin{array}{l}25,61 \\
25,24\end{array}$ & $\begin{array}{l}8,17 \\
7,84\end{array}$ & $-0,61$ & 0,542 & $-0,04$ \\
\hline Afectividad positiva & $\begin{array}{l}\text { Sí } \\
\text { No }\end{array}$ & $\begin{array}{l}2,86 \\
2,83\end{array}$ & $\begin{array}{l}0,86 \\
0,83\end{array}$ & $-0,50$ & 0,614 & $-0,03$ \\
\hline Afectividad negativa & $\begin{array}{l}\text { Sí } \\
\text { No }\end{array}$ & $\begin{array}{l}2,28 \\
2,25\end{array}$ & $\begin{array}{l}0,79 \\
0,77\end{array}$ & $-0,49$ & 0,622 & $-0,03$ \\
\hline Sintomatología depresiva & $\begin{array}{l}\text { Sí } \\
\text { No }\end{array}$ & $\begin{array}{l}10,89 \\
10,64\end{array}$ & $\begin{array}{l}4,30 \\
3,91\end{array}$ & $-0,80$ & 0,423 & $-0,06$ \\
\hline Ideación suicida & $\begin{array}{l}\text { Sí } \\
\text { No } \\
\end{array}$ & $\begin{array}{l}7,08 \\
6,50\end{array}$ & $\begin{array}{l}3,42 \\
2,82 \\
\end{array}$ & $-0,49$ & 0,513 & $-0,05$ \\
\hline
\end{tabular}

Por otra parte, en cuanto a las diferencias significativas con respecto al ejercicio físico diario, como muestra la Tabla 4, las personas que sí realizaban ejercicio físico a diario mostraron diferencias significativamente más altas en las puntuaciones medias de la afectividad positiva $(t=-4,59 ; p<0,001)$ y diferencias significativamente menores en las puntuaciones medias de la afectividad negativa $(t=$ $2,51 ; p=0,033)$ y de sintomatología depresiva $(t=3,41 ; p<0,001)$ comparativamente con aquellos participantes que no realizaron ejercicio físico diariamente durante el confinamiento domiciliario. El tamaño del efecto de la magnitud de las diferencias de las puntuaciones medias en la afectividad positiva fue moderado-bajo $(d=0,33)$, mientras que la magnitud de las diferencias de las puntuaciones medias de la afectividad negativa fue bajo $(d=0,15)$. Por último, el tamaño del efecto de la magnitud de las diferencias de las puntuaciones medias en la sintomatología depresiva fue moderado-bajo $(d=0,24)$. Estos resultados apoyan la hipótesis inicial que indicaba que los participantes del estudio que hubiesen realizado ejercicio físico diario durante el confinamiento domiciliario tendrían mejores indicadores de estado de ánimo (H2).

Tabla 4

Diferencias significativas entre personas que realizan y no realizan ejercicio físico diario en las puntuaciones medias

\begin{tabular}{|c|c|c|c|c|c|c|}
\hline & Ejercicio físico diario & Media & Desviación típica & $T$ & $p$ & $d$ \\
\hline Extensión confinamiento & $\begin{array}{l}\text { Sí } \\
\text { No }\end{array}$ & $\begin{array}{l}25,62 \\
25,03\end{array}$ & $\begin{array}{l}7,97 \\
7,93\end{array}$ & $-1,00$ & 0,314 & $-0,07$ \\
\hline Afectividad positiva & $\begin{array}{l}\text { Sí } \\
\text { No }\end{array}$ & $\begin{array}{l}2,96 \\
2,68\end{array}$ & $\begin{array}{l}0,83 \\
0,83\end{array}$ & $-4,59$ & $<0,001$ & $-0,33$ \\
\hline Afectividad negativa & $\begin{array}{l}\text { Sí } \\
\text { No }\end{array}$ & $\begin{array}{l}2,21 \\
2,34\end{array}$ & $\begin{array}{l}0,75 \\
0,81\end{array}$ & 2,13 & 0,033 & 0,15 \\
\hline Sintomatología depresiva & $\begin{array}{l}\text { Si } \\
\text { No }\end{array}$ & $\begin{array}{l}10,30 \\
11,30\end{array}$ & $\begin{array}{l}3,72 \\
4,39\end{array}$ & 3,41 & $<0,001$ & 0,24 \\
\hline Ideación suicida & $\begin{array}{l}\text { Sí } \\
\text { No }\end{array}$ & $\begin{array}{l}6,63 \\
6,79\end{array}$ & $\begin{array}{l}2,98 \\
3,14\end{array}$ & 0,73 & 0,462 & 0,05 \\
\hline
\end{tabular}

Como se puede observar en la Tabla 5, la correlación entre la extensión del confinamiento y la sintomatología depresiva no fue significativa. No obstante, los resultados de relación entre la extensión del confinamiento y la ideación suicida mostraron una correlación significativamente positiva $(r=0,07)$. Además, mientras sacar a pasear al perro no se relacionó significativamente con ninguna variable, el ejercicio físico diario mostró correlaciones significativas y positivas con la afectividad positiva $(r=0,164)$, y negativas con la afectividad negativa $(r=-0,077)$ y la sintomatología depresiva $(r=-0,123)$. Por su parte, la afectividad positiva se relacionó de forma significativa y negativa con la sintomatología depresiva $(r=-0,325)$ y la ideación suicida $(r=-0,139)$. Por el contrario, la afectividad negativa se relacionó de forma significativa y positiva con la sintomatología depresiva $(r=0,423)$ y la ideación suicida $(r=0,223)$. De manera similar, la sintomatología depresiva se relacionó de forma significativa y positiva con la ideación suicida $(r=0,543)$. Por último, en la diagonal de la Tabla 5 se muestran los índices de fiabilidad de los instrumentos.

Los presentes resultados respaldan la hipótesis inicialmente planteada sobre la existencia de relaciones significativas entre la práctica de ejercicio físico diario, el estado de ánimo, la sintomatología depresiva y la ideación suicida $(\mathrm{H} 3)$. 
Tabla 5

Tabla de correlaciones

\begin{tabular}{lccccccc}
\hline & 1 & 2 & 3 & 4 & 5 & 6 & 7 \\
\hline 1. Extensión confinamiento & - & & & & & & \\
2. Sacar a pasear al perro & 0,022 & - & & & & & \\
3. Ejercicio fisico diario & 0,036 & 0,035 & - & & & & \\
4. Afectividad positiva & $-0,003$ & 0,018 & $0,164^{* * *}$ & 0,874 & & & \\
5. Afectividad negativa & 0,058 & 0,018 & $-0,077^{*}$ & 0,048 & 0,846 & & \\
6. Sintomatología depresiva & 0,056 & 0,029 & $-0,123^{* * *}$ & $-0,325^{* * *}$ & $0,423^{* * *}$ & 0,882 & \\
7. Ideación suicida & $0,071^{*}$ & 0,050 & $-0,027$ & $-0,139^{* * *}$ & $0,223^{* * *}$ & $0,543^{* * *}$ & 0,923 \\
\hline
\end{tabular}

Nota. ${ }^{*} p<0,05,{ }^{* *} p<0,01,{ }^{* \star *} p<0,001$. Los índices de la diagonal muestran los resultados de la fiabilidad del instrumento.

\section{Ecuaciones estructurales}

Como se muestra en la Figura 2, el modelo incluido en el análisis SEM incluyó ocho variables observables (sexo como variable control), seis errores asociados a las variables, así como una covariación entre los errores de la afectividad positiva y negativa. Los resultados mostraron un ajuste apropiado del modelo: $X^{2} / d f(1, N=764)=0,615, p=0,433 ; R M S E A=0,01 ; C F I=0,99$. El modelo final tiene una suma de efectos directos e indirectos que explican un $32 \%$ de la varianza de la sintomatología depre- siva y un $31 \%$ de la varianza de la ideación suicida. Las relaciones directas significativas mostraron que el ejercicio físico diario se asoció positivamente en la afectividad positiva $(\beta=0,17)$, y los días de confinamiento se asociaron positivamente con la sintomatología depresiva $(\beta=0,07)$. Asimismo, la afectividad positiva se asoció negativamente con la sintomatología depresiva $(\beta=-0,34)$, mientras que la afectividad negativa lo hizo de forma positiva $(\beta=0,47)$. Por último, la sintomatología depresiva se asoció positivamente con la ideación suicida $(\beta=0,58)$.

De manera indirecta, la variable días de confinamiento correlacionó positiva y significativamente con la sintomatología depresiva $(\beta=0,02)$ y la ideación suicida $(\beta=0,05)$, mientras que realizar ejercicio físico diario correlacionó negativamente tanto con la sintomatología depresiva $(\beta=-0,09)$ como con la ideación suicida $(\beta=-0,07)$. Por último, la afectividad positiva y la negativa se asociaron significativamente con los niveles de ideación suicida de forma negativa $(\beta=-0,26)$ y positiva $(\beta=0,20)$, respectivamente. Estos resultados respaldan parcialmente la hipótesis inicialmente planteada sobre la realización de ejercicio físico diario y sacar a pasear al perro durante la extensión del confinamiento mejora el estado de ánimo y disminuye la sintomatología depresiva y la ideación suicida $(\mathrm{H} 4)$.

Figura 2

Modelo estimado de relación causal

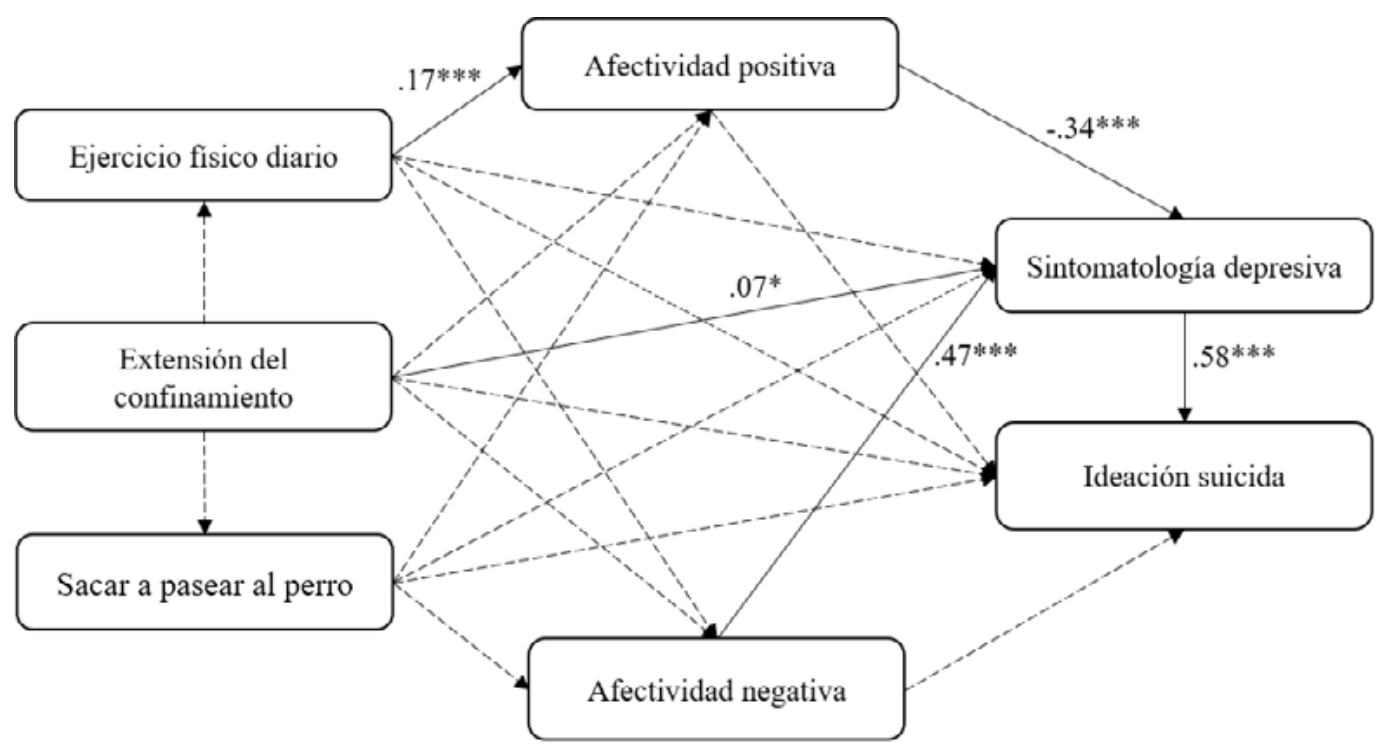

La prueba de invariancia por sexos del modelo (Byrne, Shavelson, \& Muthén, 1989) mostró ausencia de diferencias significativas entre hombres-mujeres en el modelo: $X^{2}(1, N=764)=1.291$, $p=0,256$. Por lo tanto, el modelo es consistente para ambos sexos. 


\section{Discusión}

Este estudio tuvo como objetivo analizar la relación de la extensión del confinamiento impuesto por el Gobierno de España en el primer estado de alarma, entre el 14 de marzo y el 20 de junio de 2020, la realización de ejercicio físico diario y la conducta de sacar a pasear al perro en el estado de ánimo, la sintomatología depresiva y la ideación suicida durante el confinamiento domiciliario.

En relación con los resultados obtenidos se puede apreciar que la extensión del confinamiento que inicialmente y de manera aislada no mostraba relación significativa con la sintomatología depresiva, en un contexto multivariante mostró una relación directa, lo cual es apoyado por la literatura (Hawryluck et al., 2004). Esto puede deberse a que la experimentación de emociones negativas de manera prolongada influye en el desarrollo de sintomatología depresiva. A su vez, los resultados han mostrado que la extensión del confinamiento se relacionó de manera directamente significativa con la ideación suicida. Sin embargo, al incluir variables del estado de ánimo como son la afectividad positiva y negativa, y la sintomatología depresiva, esta relación directa desapareció. Estos resultados muestran los mecanismos psicológicos de acción de los estados de ánimo en la salud mental durante un estado de privación de libertad (Brooks et al, 2020; Hawryluck et al, 2004; Marjanovic et al., 2007; Reynolds et al, 2008). Estos mecanismos de acción pueden dar lugar a pensamientos rumiativos durante y después del confinamiento y la pandemia a largo plazo. Por lo tanto, la extensión de días sin salir del domicilio parece repercutir en un aumento de emociones negativas (Lee et al., 2005; Rosselló \& Hernández, 2004).

Por otro lado, la práctica de ejercicio físico se relacionó de manera significativa con el estado de ánimo, reduciendo los estados emocionales negativos y aumentando los estados emocionales positivos (Jiménez et al., 2008). Estos resultados indican que la realización de ejercicio físico diario podría servir como una evasión de estados emocionales negativos, así como proporcionar un aumento y prolongación de la experimentación de las emociones positivas (Biddle et al., 2000; Zach, Zeev, Ophir, \& Eilat-Adar, 2021). En la misma línea, el ejercicio físico diario se relacionó con la sintomatología depresiva de manera negativa (Lawlor \& Hopker, 2001), lo que podría deberse a que el ejercicio físico actuaría como factor protector del estado de ánimo negativo y éste afectaría de manera directa a la sintomatología depresiva. Esta idea va en línea con los resultados encontrados en el análisis multivariante, en el que el ejercicio físico no se relacionó de manera directa con la sintomatología depresiva sino de manera indirecta a través del estado de ánimo. Igualmente, la realización de ejercicio físico a diario se relacionó directamente con la ideación suicida y la sintomatología depresiva de manera negativa, pero posteriormente, en el análisis multivariante, se comprobó que dicha relación era indirecta a través de la afectividad positiva y negativa (Alzate Pérez et al., 2013; González-Forteza et al., 2000). Estos resultados contradicen los derivados del análisis de diferencias que no mostraron diferencias significativas en las puntuaciones medias de ideación suicida entre los participantes que realizaron ejercicio físico diario y aquellos que no lo realizaron (González-Forteza et al., 2000), e indican la necesidad de entender de manera conjunta la influencia de mecanismos psicológicos en el desarrollo positivo del estado de ánimo y la prevención de la patología (Siabato Macías et al., 2017).

Por el contrario, la realización de la conducta de sacar a pasear al perro ha mostrado no ha mostrado asociarse con los estados de ánimo de los participantes, lo cual contradice investigaciones previas (Balazote, 1999; Wilson, 1998). Estos resultados pueden deberse a que durante el periodo de confinamiento las personas que no tuviesen perro al que pasear dispusieran de otro tipo de evasión ante la privación de libertad (Oria et al., 2020) y por lo tanto no mostrasen peores estados de ánimo (Gutiérrez et al., 2007).

\section{Limitaciones}

El presente estudio presenta ciertas limitaciones. En primer lugar, puesto que se ha evaluado mediante una escala autoaplicada exclusivamente, algunos de los datos pueden estar sesgados al basarse en la percepción personal de casa sujeto. Por otro lado, existen multitud de variables atenuantes que pueden influir en la sintomatología depresiva, como por ejemplo si las personas trabajaban, vivían solas, el tipo de ejercicio físico realizado, etc., que no se han evaluado en este estudio. Futuros estudios podrían analizar el papel de dichas variables en este tipo de situaciones de confinamiento y su influencia en la sintomatología depresiva.

A pesar de las limitaciones, este estudio tiene una serie de fortalezas. El tamaño de la muestra de participantes del estudio permite aplicar los resultados a la población general. Además, debido a la ausencia de investigaciones sobre el tema, este estudio aporta información relevante sobre cómo afecta la situación de confinamiento por el estado de alarma en España a partir del 14 de marzo en el aspecto psicológico. De los resultados derivados de este trabajo cabe realizar algunas recomendaciones. En primer lugar, aunque es cierto que hay un número considerable de estudios sobre cómo afecta 
el ejercicio físico a nivel psicológico, no se ha encontrado una amplia literatura sobre el efecto del ejercicio físico en las ideaciones suicidas. Por otro lado, la literatura sobre la afectación del confinamiento es muy limitada y la mayoría de éstas están referidas a ambientes carcelarios, por lo tanto, el estudio del confinamiento en la población general es un campo aún por investigar.

Teniendo en cuenta los efectos negativos que se pueden producir en algunas personas en situación de confinamiento, se podrían crear programas específicos tanto de prevención como como de tratamiento para reducir el impacto psicológico de estados temporales de privación de libertad. $Y$ enriquecer la intervención con el ejercicio físico regular.

\section{Conclusiones}

Los efectos del periodo de cuarentena a causa de una pandemia pueden ser duraderos, como evitar volver a la normalidad hasta varios meses más tarde; en otros casos, la afectación puede ser permanente (Cava, Fay, Beanlands, McCay, \& Wignall, 2005). En consecuencia, los datos obtenidos en el presente estudio pretenden ser de utilidad para conocer los efectos que pudiese provocar la situación de confinamiento durante el estado de alarma del 14 de marzo de 2020 a nivel psicológico. Además, esto podría ser utilizado desde un punto de vista preventivo ante posibles situaciones similares.

Referencias
1. Akandere, M., \& Tekin, A. (2005). Efectos del ejercicio físico sobre la ansiedad. Publice Standard.

2. Alzate Pérez, L. J., Betancur Betancur, M., Castaño Castrillón, J. J., Constanza Cañón, S., Castellanos Sánchez, P. L., Guerrero González, J., ... \& Tique Estrada, J. E. (2013). Factor de riesgo suicida según dos cuestionarios, y factores asociados en estudiantes de la Universidad Nacional de Colombia sede Manizales. Colombia Press.

3. Arsenault-Lapierre, G., Kim, C., \& Turecki, G. (2004). Psychiatric diagnoses in 3275 suicides: a meta-analysis. BMC Psychiatry, 4(1), 1-11. https://doi.org/10.1186/1471-244x-4-37

4. Bados, A., Solanas, A., \& Andrés, R. (2005). Psychometric properties of the Spanish version of depression, anxiety and stress scales (DASS). Psicothema, 17(4), 679-683.

5. Balazote, P. (1999). Animales de compañía y mejora de las relaciones interpersonales en un centro geriátrico. In Fundación Purina (Ed.), Animales de compañía fuente de salud. Comunicaciones IV Congreso Internacional (pp. 45-55). Barcelona: Fundación Purina.

6. Biddle, S. J. H., Fox, K. R., \& Boutcher, S. H. (2000). Physical activity and psychological well-being. Londres: Routledge.

7. Blum, R. W., Halcón, L., Beuhring, T., Pate, E., Campell-Forrester, S., \& Venema, A. (2003). Adolescent health in the Caribbean: risk and protective factors. American Journal of Public Health, 93(3), 456-460.

8. Brooks, S. K., Webster, R. K., Smith, L. E., Woodland, L., Wessely, S., Greenberg, N., \& Rubin, G. J. (2020). The psychological impact of quarantine and how to reduce it: rapid review of the evidence. The Lancet, 395, 912-920. https://doi.org/10.1016/s0140-6736(20)30460-8

9. Brown, J., Cohen, P., Johnson, J. G., \& Smailes, E. M. (1999). Childhood abuse and neglect: specificity of effects on adolescent and young adult depression and suicidality. Journal of the American Academy of Child \& Adolescent Psychiatry, 38(12), 1490-1496. https://doi.org/10.1097/00004583199912000-00009

10. Buz, J., Pérez-Arechaederra, D., Fernández-Pulido, R., \& Urchaga, D. (2015). Factorial structure and measurement invariance of the PANAS in Spanish older adults. The Spanish Journal of Psychology, 18, 1-11. https://doi.org/10.1017/sjp.2015.6

11. Castro-Rueda, V. A., Martínez-Villalba, A. M. R., Camacho, P. A., \& Rueda-Jaimes, G. E. (2010). Factores de riesgo y protectores para intento suicida en adultos colombianos con suicidabilidad. Revista Colombiana de Psiquiatría, 39(4), 705-715. https://doi.org/10.1016/s00347450(14)60210-9

12. Cava, M. A., Fay, K. E., Beanlands, H. J., McCay, E. A., \& Wignall, R. (2005). The experience of quarantine for individuals affected by SARS in Toronto. Public Health Nursing, 22(5), 398-406. https://doi.org/10.1111/j.0737-1209.2005.220504.x

13. Cavanagh, J. T., Carson, A. J., Sharpe, M., \& Lawrie, S. M. (2003). Psychological autopsy studies of suicide: a systematic review. Psychological Medicine, 33(03), 395-405. https://doi.org/10.1017/ s0033291702006943

14. Chang, E. C., \& Chang, O. D. (2016). Development of the Frequency of Suicidal Ideation Inventory: Evidence for the validity and reliability of a brief measure of suicidal ideation frequency in a college student population. Cognitive Therapy and Research, 40(4), 549-556. https://doi. org/10.1007/s10608-016-9758-0 
15. Chen, F. F., Jing, Y., Hayes, A., \& Lee, J. M. (2013). Two concepts or two approaches? A bifactor analysis of psychological and subjective well-being. Journal of Happiness Studies, 14(3), 10331068. https://doi.org/10.1037/e578192014-355

16. Cohen, J. (1988). Statistical Power Analysis for the Behavioral Sciences. 2nd. edit., Hillsdale, N.J., Erlbaum (primera edición, 1977 New York: Academic Press).

17. Granados, S. H. B., \& Cuéllar, Á. M. U. (2018). Influencia del deporte y la actividad física en el estado de salud físico y mental: una revisión bibliográfica. Katharsis: Revista de Ciencias Sociales, (25), 141-160.

18. De la Torre López, J., Costillas, L. P., \& Sánchez-Álvarez, N. (2016). Análisis y diseño intervencionista de la conducta suicida en población con dificultades socio-económicas: enfoque preventivo desde la Psicología Positiva. Documentos de Trabajo Social: Revista de Trabajo y Acción Social, (57), 100-124.

19. De Leo, D., Scocco, P., Marietta, P., Schmidtke, A., Bille-Brahe, U., Kerkhof, A. J., ... \& Michel, K. (1999). Physical illness and parasuicide: evidence from the European Parasuicide Study Interview Schedule (EPSIS/WHO-EURO). The International Journal of Psychiatry in Medicine, 29(2), 149-163. https://doi.org/10.2190/e87k-fg03-chee-ujd3

20. DiGiovanni, C., Conley, J., Chiu, D., \& Zaborski, J. (2004). Factors influencing compliance with quarantine in Toronto during the 2003 SARS outbreak. Biosecurity and Bioterrorism: Biodefense Strategy, Practice, and Science, 2(4), 265-272. https://doi.org/10.1089/bsp.2004.2.265

21. Fariñas, T. (2020). La cuarentena, más allá de España: casi el $40 \%$ de la población mundial, bajo encierro. mayo 14, 2020, de El Confidencial Sitio web: https://www.elconfidencial.com/ mundo/2020-03-28/mapa-cuarentenas-mundo-paises-coronavirus 2521831/

22. Foster, T., Gillespie, K., \& McClelland, R. (1997). Mental disorders and suicide in Northern Ireland. The British Journal of Psychiatry, 170(5), 447-452. https://doi.org/10.1192/bjp.170.5.447

23. Gutiérrez, G., Granados, D. R., \& Piar, N. (2007). Interacciones humano-animal: características e implicaciones para el bienestar de los humanos. Revista Colombiana de Psicología, 16(1), 163-184.

24. Hawryluck, L., Gold, W. L., Robinson, S., Pogorski, S., Galea, S., \& Styra, R. (2004). SARS control and psychological effects of quarantine, Toronto, Canada. Emerging Infectious Diseases, 10(7), 1206-1212. https://doi.org/10.3201/eid1007.030703

25. Huertas, F., López, A. L., Pablos, A., Colado, J. C., Pablos-Abella C., \& Campos, J. (2003). Efectos de un programa de ejercicio físico sobre el bienestar psicológico de mujeres mayores de 55 años. Revista de Psicología del Deporte, 12, 7-26.

26. Jeong, H., Yim, H. W., Song, Y. J., Ki, M., Min, J. A., Cho, J., \& Chae, J. H. (2016). Mental health status of people isolated due to Middle East Respiratory Syndrome. Epidemiology and Health, 38, 137-144. https://doi.org/10.4178/epih.e2016048

27. Jiménez, M. G., Martínez, P., Miró, E., \& Sánchez, A. I. (2008). Bienestar psicológico y hábitos saludables: ¿están asociados a la práctica de ejercicio físico? International Journal of Clinical and Health Psychology, 8(1), 185- 202.

28. Lawlor, D. A., \& Hopker, S. W. (2001). The effectiveness of exercise as an intervention in the management of depression: Systematic review and metaregression analysis of randomised controlled trials. British Medical Journal, 322, 763-767. https://doi.org/10.1136/bmj.322.7289.763

29. Lee, S., Chan, L. Y., Chau, A. M., Kwok, K. P., \& Kleinman, A. (2005). The experience of SARS-related stigma at Amoy Gardens. Social Science \& Medicine, 61(9), 2038-2046. https://doi. org/10.1016/..socscimed.2005.04.010

30. Lovibond, P. F., \& Lovibond, S. H. (1995). The structure of negative emotional states: Comparison of the Depression Anxiety Stress Scales (DASS) with the Beck Depression and Anxiety Inventories. Behaviour Research and Therapy, 33(3), 335-343. https://doi.org/10.1016/00057967(94)00075-u

31. Marjanovic, Z., Greenglass, E. R., \& Coffey, S. (2007). The relevance of psychosocial vari- ables and working conditions in predicting nurses' coping strategies during the SARS crisis: an online questionnaire survey. International Journal of Nursing Studies, 44(6), 991-998. https://doi. org/10.1016/j.ijnurstu.2006.02.012

32. Oria, Ó. G., Carricajo, P. B., \& Rivas, V. G. (2020). Cumplimiento del confinamiento por COVID-19 en España: una aproximación. In Pensamientos sociales desde la nueva realidad (pp. 139-158). AnthropiQa.

33. Qin, P., \& Mortensen, P. B. (2003). The impact of parental status on the risk of completed suicide. Archives of General Psychiatry, 60(8), 797-802. https://doi.org/10.1001/archpsyc.60.8.797 
34. Real Decreto $463 / 2020$, de 14 de marzo, estado de alarma por crisis sanitaria ocasionada por el COVID-19 (2020). Boletín Oficial del estado, 67.

35. Reynolds, D. L., Garay, J. R., Deamond, S. L., Moran, M. K., Gold, W., \& Styra, R. (2008). Understanding, compliance and psychological impact of the SARS quarantine experience. Epidemiology \& Infection, 136(7), 997-1007. https://doi.org/10.1017/s0950268807009156

36. Rosselló, J., \& Hernández, M. N. B. (2004). Ideación suicida, depresión, actitudes disfuncionales, eventos de vida estresantes y autoestima en una muestra de adolescentes puertorriqueños/ as. Interamerican Journal of Psychology, 38(2), 295-302.

37. Sánchez-Álvarez, N., Extremera-Pacheco, N., Rey, L., Chang, E. C., \& Chang, O. D. (2020). Frequency of Suicidal Ideation Inventory: Psychometric Properties of the Spanish Version. Psicothema, 32(2), 253-260. https://doi.org/10.1037/t80124-000

38. Seligman, M. E., \& Csikszentmihalyi, M. (2000). Positive psychology: An introduction. American Psychological Association.

39. Siabato Macías, E. F., Forero Mendoza, I. X., \& Salamanca Camargo, Y. (2017). Asociación entre depresión e ideación suicida en un grupo de adolescentes colombianos. Pensamiento Psicológico, 15(1), 51-61.

40. Thacore, V. R., \& Varma, S. L. (2000). A study of suicides in Ballarat, Victoria, Australia. Crisis: The Journal of Crisis Intervention and Suicide Prevention, 21(1), 26-31. https://doi.org/10.1027//02275910.21 .1 .26

41. Watson, D., \& Clark, L. A. (1984). Negative affectivity: the disposition to experience aversive emotional states. Psychological Bulletin, 96(3), 465-490. https://doi.org/10.1037/0033-2909.96.3.465

42. Watson, D., Clark, L. A., \& Tellegen, A. (1988). Development and validation of brief measures of positive and negative affect: the PANAS scales. Journal of Personality and Social Psychology, 54(6), 1063. https://doi.org/10.1037/0022-3514.54.6.1063

43. Wheeler, A. R., Shanine, K. K., Leon, M. R., \& Whitman, M. V. (2014). Student-recruited samples in organisational research: A review, analysis, and guidelines for future research. Journal of Occupational and Organizational Psychology, 87, 1-26. https://doi.org/10.1111/joop.12042

44. Wilson, C. C. (1998). Companion animals in human health. Sage.

45. World Health Organization-WHO (2014). Preventing suicide: a global imperative. Washington, DC: Panamerican Health Organization.

46. Yoon, M. K., Kim, S. Y., Ko, H. S., \& Lee, M. S. (2016). System effectiveness of detection, brief intervention and refer to treatment for the people with post-traumatic emotional distress by MERS: a case report of community-based proactive intervention in South Korea. International Journal of Mental Health Systems, 10(1), 1-5. https://doi.org/10.1186/s13033-016-0083-5

47. Zach, S., Zeev, A., Ophir, M., \& Eilat-Adar, S. (2021). Physical activity, resilience, emotions, moods, and weight control of older adults during the COVID-19 global crisis. European Review of Aging and Physical Activity, 18(1), 1-8. https://doi.org/10.1186/s11556-021-00258-w

RECIBIDO: 6 de mayo de 2021

MODIFICADO: 16 de noviembre de 2021

ACEPTADO: 16 de noviembre de 2021 